\title{
Interpretación medicolegal para el abordaje de individuos con desórdenes del desarrollo sexual en Colombia
}

\section{Interpretation of medical-legal Colombian legislation to treat individuals with disorders of sexual development} \author{
Catalina Forero $^{5}$ Camilo Orjuela $^{6}$ Fernando Suarez $^{7}$ \\ ${ }^{1}$ Seattle Children's Hospital, University of Washington, Seattle, \\ United States \\ ${ }^{2}$ Department of Urology, Fundacion Santa Fe De Bogota, Andes \\ University, Bogota, Colombia \\ ${ }^{3}$ Comité de Ética en Investigación con Seres Humanos, Riesgo y \\ fractura S. A., Cayre, Universidad Javeriana, Bogota, Colombia \\ ${ }^{4}$ Division of Urology, Hospital Universitario San Ignacio, Pontificia \\ Universidad Javeriana, Bogota, Colombia \\ ${ }^{5}$ Division of Pediatric Endocrinology, Hospital Universitario San \\ Ignacio, Pontificia Universidad Javeriana, Bogota, Colombia \\ 6 Pediatric Urology, Universidad Nacional, Bogota, Colombia \\ ${ }^{7}$ Instituto de Genetica Humana, Pontificia Universidad Javeriana, \\ Bogota, Colombia
}

Nicolas Fernandez ${ }^{1}$ Laura Zuluaga ${ }^{2}$ Maria Fernanda Perez ${ }^{3}$ Jaime Perez ${ }^{2,4}$ Camila Cespedes $^{5}$

Urol Colomb 2021;30(4):e240-e252.

Dirección para correspondencia Nicolas Fernandez, M.D., PhD,
Seattle Children's Hospital, University of Washington, Seattle,
United States (e-mail: nicolas.fernandez@seattlechildrens.org).

\section{Resumen}

Palabras clave

- desórdenes del desarrollo sexual

- legal

- cirugía de reafirmación de género
Objetivo Los desórdenes del desarrollo sexual (DDSs) son un grupo de condiciones médicas cuyo manejo implica un enfoque transdisciplinario. En la legislación colombiana, no existe una regulación específica en materia de intervenciones médicas en individuos con DDSs. La Corte Constitucional Colombiana se ha encargado de proferir sentencias, en las cuales se han establecido unos lineamientos y parámetros para el manejo jurídico de los casos de ambigüedad genital.

Teniendo en cuenta lo anterior, nos proponemos en este artículo exponer y analizar, desde la visión de un grupo transdisciplinario, las sentencias más relevantes que han sido proferidas por la Corte Constitucional Colombiana.

Métodos Se realizó una revisión narrativa en las bases de datos de la Corte Constitucional y de la literatura legal. Se recopilaron todas las sentencias disponibles, y se evaluaron lo casos clínicos identificados por el grupo transdisciplinario de DDSs del Hospital Universitario San Ignacio. Finalmente, los autores generaron por consenso un texto de discusión, como guía para los lectores, luego de analizar cada una de las sentencias y los casos presentados.

Resultados Se identificaron 9 sentencias y los antecedentes que llevaron a la elaboración de cada una de ellas. Los nueve casos presentados contienen conceptos importantes para la articulación por parte del grupo transdisciplinario, tales como, recibido

25 de septiembre de 2020

aceptado

19 de agosto de 2021
DOI https://doi.org/

$10.1055 / \mathrm{s}-0041-1740376$.

ISSN 0120-789X.

e ISSN 2027-0119. (c) 2021. Sociedad Colombiana de Urología. All rights reserved. This is an open access article published by Thieme under the terms of the Creative Commons Attribution-NonDerivative-NonCommercial-License, permitting copying and reproduction so long as the original work is given appropriate credit. Contents may not be used for commercial purposes, or adapted, remixed, transformed or built upon. (https://creativecommons.org/ licenses/by-nc-nd/4.0/)

Thieme Revinter Publicações Ltda., Rua do Matoso 170, Rio de Janeiro, RJ, CEP 20270-135, Brazil 
Abstract

\section{Keywords}

- disorders of sexual development

- legal

- gender-affirming surgery autonomía, consentimiento sustituto y asistido, e indicación médica del tratamiento. Se excluyeron las sentencias T-692/99 y la T-918/2012, dado que daban información duplicada con referencia a sentencias incluidas en este documento.

Conclusión La Corte Constitucional Colombiana ha proferido sentencias en las cuales no se ha contado con la participación de grupos de expertos. La terminología usada no describe apropiadamente el lenguaje técnico que se aplica al manejo de nuestros pacientes. Para el abordaje de individuos con DDSs, resulta muy importante conocer la relevancia de las sentencias basadas en un análisis individual de cada caso.

Objective Disorders of sexual development (DSDs) are a group of conditions that require a transdisciplinary approach. In the Colombian legislation, there are no specific regulations regarding medical interventions in individuals with DSDs. The Colombian Constitutional Court has issued sentences establishing some guidelines and parameters for the legal management of cases of genital ambiguity.

Considering the aforementioned information, the present manuscript aims to describe and analyze, from the perspective of a transdisciplinary team, the most relevant sentences issued by the Colombian Constitutional Court.

Methods We performed a narrative review of the database of the Colombian Constitutional Court and of the legal literature. We compiled all available court sentences and evaluated the clinical cases identified by the transdisciplinary team at Hospital Universitario San Ignacio. Lastly, the authors composed, through consensus, a text for discussion, as a guide to the readers, after the analysis of each of the sentences and cases presented.

Results We identified nine sentences and the precedents that led to their development. The nine cases presented contain important concepts for the work of the transdisciplinary team, such as autonomy, substitute and assisted consent, and the medical indication of treatment. Sentences T-692/99 and T-918/2012 were excluded, as they provided duplicate information concerning sentences included in this document.

Conclusion The Colombian Constitutional Court has issued sentences without the participation of a team of experts in DSDs. The terminology used does not properly describe the jargon that is applied in the management of our patients. In order to approach individuals with DSDs, awareness regarding the relevance of the sentences based on an individual analysis of each case is of great importance.

\section{Introducción}

Los desórdenes del desarrollo sexual (DDSs) son un grupo de condiciones médicas cuyo manejo implica un enfoque transdisciplinario. $^{1}$ En la actualidad, los grupos transdisciplinarios ofrecen un abordaje integral a los pacientes y sus familias, y procuran tanto el bienestar inmediato como a largo plazo de los individuos afectados. ${ }^{2}$

La gran complejidad que involucra el género anatómico, fisiológico, la orientación y la identidad de género, y los diferentes procesos psicológicos y sociales que interaccionan entre cada uno de estos ejes hace muy compleja la aproximación de estas condiciones.

El tratamiento de pacientes en pediatría, a diferencia de otras áreas de la medicina, tiene unas condiciones especiales que involucran, en la toma de decisiones, la participación de terceros. Es por esto que el consentimiento es un proceso más complejo, teniendo en cuenta que son los representantes legales quienes toman, en la mayoría de las veces, decisiones por el paciente sin que él tenga la oportunidad de participar en dicho proceso. Adicionalmente, los efectos de los procedimientos realizados en la infancia tienen efectos a largo plazo. Puntualmente en los DDSs, los procedimientos realizados involucran áreas de altísima complejidad, con impacto en los ejes fenotípico, psicosexual, y de fertilidad. ${ }^{3,4}$

A nivel mundial, existe un continuo crecimiento y preocupación acerca de la realización de cirugías descritas por algunos como mutilantes o irreversibles en individuos con DDSs. ${ }^{5}$ Aunque aún falta mucho por entender, es claro que los conflictos actuales giran en torno a resultados de tratamientos que no se practican en la actualidad. Adicionalmente, parte de las razones por las cuales es mediáticamente llamativo este tema de discusión es por la falta de unanimidad en la terminología usada, que involucra 
múltiples condiciones en un mismo grupo. El claro ejemplo es incluir las condiciones de disforia de género con los DDSs toda vez que existen diferencias concretas entre uno y otro. Un grupo significativo de pacientes con DDSs se caracteriza por presentar genitales ambiguos al momento del nacimiento, es decir, cuando los órganos sexuales externos no son completamente masculinos ni femeninos. Esto se debe a las anormalidades en los cromosomas/genes involucrados en el desarrollo gonadal y genital y su interacción ambiental, entre otras circunstancias. ${ }^{6}$

Ahora bien, en la disforia de género se eliminó la condición de DDSs, por considerarse que no se debe incluir en la misma categoría, dadas las diferencias que presentan.

La identidad de género se entiende como una construcción subjetiva que lleva a cabo cada persona sobre su identificación como hombre, mujer, persona de género neutro, de género fluido, trans, sin género, entre otras. De esta manera, la disforia de género es aquella situación en la que las personas no se identifican con el sexo/género asignado al nacer, y transitan al otro género. No obstante, los casos de individuos con DDSs pueden presentar disforia de género, porque el substrato biológico y el entorno emocional son más complejos, dado que involucran una alteración en la formación genital y de los órganos reproductivos, situación que no es del todo cierta en aquellos con disforia de género, quienes tienen genitales formados correctamente en cuanto a su potencial funcional.

En la legislación colombiana, no existe una regulación específica en materia de intervenciones médicas en individuos con DDSs; se encuentran normas del código civil que establecen lo relacionado con la capacidad de toma de decisiones en menores de edad, pero que no resultan suficientes a la hora de abordar casos concretos en materia de DDSs; no obstante, la Corte Constitucional Colombiana se ha encargado de proferir sentencias, tanto de tutela como de unificación, por las cuales se han establecido unos lineamientos y parámetros para el manejo jurídico de los casos de ambigüedad genital.

Teniendo en cuenta lo anterior, nos proponemos en este artículo exponer y analizar, desde la visión de un grupo transdisciplinario, las sentencias más relevantes que han sido proferidas por la Corte Constitucional, en virtud de las cuales se han establecido diferentes criterios aplicables a los casos de DDSs que se presentan en nuestra práctica clínica diaria, con el objetivo de tomarlos como guía para los profesionales de la salud en el proceso de atención a este tipo de pacientes.

\section{Métodos}

Se llevó a cabo una revisión de la literatura en las bases de datos de la Corte Constitucional, tomando la muestra de las sentencias relacionadas con DDSs que ha proferido la corte. ${ }^{7}$ Los términos de búsqueda usados fueron: ambigüedad genital, intersexualidad, principio de autonomía, personalidad jurídica del niño, y indeterminación sexual. Se trazó una línea del tiempo desde el año 1995 al 2014, escogiendo el primer fallo y el más reciente como límites temporales, y se hizo énfasis en sentencias hito que han cambiado la línea jurisprudencial en diferentes aspectos en la Corte Constitucional respecto de la materia objeto de reflexión. Una vez recopiladas todas las sentencias disponibles, se hizo un análisis de la sentencia con base en la experiencia del grupo transdisciplinario de DDSs del Hospital Universitario San Ignacio, en Bogotá, compuesto por Urología Pediátrica, Endocrinología Pediátrica, Cirugía Pediátrica, Psiquiatría Infantil, Trabajo Social, y Genética, para facilitar la interpretación de la jurisprudencia aplicada a la práctica clínica. Finalmente, los autores generaron por consenso unas conclusiones, como guía para los lectores, luego de analizar cada una de las sentencias y los casos presentados.

\section{Resultados}

Se identificaron nueve sentencias que se presentan a continuación, así como los antecedentes que llevaron a la elaboración de cada una de ellas. Los nueve casos presentados contienen conceptos importantes para la articulación por parte del grupo transdisciplinario, tales como autonomía, consentimiento sustituto y asistido, e indicación médica del tratamiento. Se excluyeron las sentencias T-692/99 y la T-918/2012, pues daban información duplicada con referencia a sentencias incluidas en este documento.

Encontramos que, en las diferentes sentencias, se utilizan términos inapropiados e inexactos, desde el punto de vista médico (como "readecuación", usado a vecespara señalar la "reasignación de sexo"), que tienen implicaciones técnicas muy importantes. Estos términos incluidos ameritan investigaciones futuras.

1. T-477, del 23 de octubre de 1995 https://www. corteconstitucional.gov.co/relatoria/1995/t-477-95.htm

Magistrado ponente: Dr.Alejandro Martínez Caballero

Hechos que llevaron a la sentencia: caso de mutilación genital. Se trata de un paciente masculino quien, a los 6 meses de edad, sufre la amputación traumática del pene y los testículos, sin que se tenga claridad del mecanismo (¿mordedura canina?; ¿herida con elemento cortopunzante?). El equipo médico que le atiende, ante la limitación de no poder reconstruir unos genitales masculinos funcionantes, sugiere una reasignación al sexo femenino, como lo describía la literatura médica predominante en ese momento por autores como Money. ${ }^{8}$

Los padres del menor eran campesinos semianalfabetos "habitantes de un área alejada y subdesarrollada", y, con sus limitantes, "dibujan su firma en un escrito que autoriza cualquier tipo de tratamiento, incluyendo el cambio de sexo, que conlleve a mejorar la situación actual" del paciente, sin que, según la sentencia, "los padres fueran conscientes de la trascendencia del "cambio de sexo". Al niño se le practicó una meatotomía a los 7,5 meses de edad, y fue entregado a una casa de monjas para que fuese criado como una niña, siendo evidente una desadaptación importante al sexo asignado ("tenía comportamientos de varón en la postura para la micción y en algunos juegos”). A 
los 5 años, se inicia el tratamiento médico-psiquiátrico para acondicionar la conciencia del menor a fin de que se adaptara como mujer, a pesar del rechazo evidente por parte del afectado. A los 6 años de edad, se hace el trámite notarial para cambiar el nombre por uno femenino. Por esta misma época, se le realiza una segunda cirugía de reasignación de genitales ( $\sin$ un nuevo consentimiento informado), que dio como resultado, según los médicos, un "fenotipo femenino". De acuerdo con el hospital, hubo "reasignación de género". Adicionalmente, al niño se le inició manejo hormonal.

Con el tiempo, el menor comenzó a manifestar inconformidad con su asignación femenina; reclamaba que se sentía niño, y expresaba: a mí me operaron cuando estaba pequeñito, me operaron la vagina, dizque, para ponerme mujer, pero uno grande ya tiene más pensamiento y decide. Yo decidí ser un hombre, porque hombre era yo desde chiquitico. Yo decidí ser hombre, porque uno es hombre como nació. [...] Por qué no esperaron a que yo estuviera grande para yo saber lo que me iban a hacer y hasta poder escoger; pero, como uno estaba chiquito, hacían lo que querían con uno. En el momento en que el niño tenía 9 años de edad, el personero del municipio presentó una tutela con el fin de proteger los derechos del menor y solicitar que fuese tratado como niño, y no como niña. De esta manera, a la Corte Constitucional le corresponde resolver lo siguiente:

Problema jurídico: ¿Fue o es legítima la conducta de las autoridades y de los particulares que participaron en el proceso de reasignación de sexo del menor de conformidad con la Constitución Política?

Análisis de la sentencia: esta es una sentencia clásica de la Corte Constitucional en la que se establecen inicialmente los elementos básicos del consentimiento en la relación médicopaciente. Se define el concepto del consentimiento informado, y se analizan de manera pormenorizada los aspectos relacionados con el consentimiento del paciente menor de edad en un escenario como el que se ha descrito. Al respecto del consentimiento del menor de edad, la corte considera que hay tres elementos que deben ser tenidos en cuenta en situaciones de esta naturaleza:

1) “La urgencia e importancia misma del tratamiento para los intereses del menor";

2) "La intensidad del impacto del tratamiento sobre la autonomía actual y futura del niño"; y

3) "La edad misma del menor", siendo evidente que es diferente la situación de un recién nacido que la de un adolescente.

Al respecto de la intensidad del impacto del tratamiento, menciona la corte que "la doctrina ha establecido una distinción [...] entre intervenciones médicas ordinarias, que no afectan el curso cotidiano de la vida del paciente, e intervenciones extraordinarias, que se caracterizan porque es 'notorio el carácter invasivo y agobiante del tratamiento médico en el ámbito de la autonomía personal', de suerte que se afecta 'de manera sustancial el principio de autodeterminación personal'. Esto incluye obviamente una ponderación de los posibles efectos irreversibles de ciertas intervenciones médicas, por cuanto los tratamientos que tienen tal carácter predeterminan, en muchos aspectos, la vida futura del menor".

Con estas consideraciones, la corte establece que "NO es posible la 'readecuación de sexo', sin la autorización directa del paciente", y deja claro que "Los niños no son propiedad de nadie: ni son propiedad de sus padres, ni son propiedad de la sociedad. Su vida y su libertad son de su exclusiva autonomía". Anota adicionalmente que "los padres no pueden permitir que se altere la IDENTIDAD (EN LO SEXUAL) DE SU HIJO”, y que "los médicos no podían basarse en esa autorización paterna para hacer el tratamiento". La sentencia describe también que, al violarse el derecho a la identidad, también se violenta el derecho a la dignidad humana y al libre desarrollo de la personalidad.

En las determinaciones de esta sentencia, se anota que "Es inhumano que, si el pene y los testículos fueron cercenados, la solución sea volver a la víctima mujer", y que "el expreso consentimiento informado del propio paciente es indispensable para cualquier tratamiento médico de readecuación del sexo". Ordena la corte al notario que mantenga en el registro civil el nombre de varón del paciente, y resuelve que el Instituto Colombiano de Bienestar Familiar debe proteger al paciente y brindarle "el tratamiento integral físico y sicológico requerido para la readecuación del menor".

La Corte envía copia de este fallo "a la Defensoría del Pueblo para que, si el menor lo acepta, se designe por el Defensor del Pueblo un abogado que inicie el correspondiente juicio de responsabilidad civil por el daño sufrido por el menor debido a la 'readecuación de sexo' o 'transformación de órganos sexuales', practicada sin su consentimiento".

Entonces, esta sentencia deja claro que las cirugías de reasignación de sexo no pueden ser realizadas sin el consentimiento del paciente, que el consentimiento sustituto de los padres no es válido en estas circunstancias, y que el no acatamiento de esta jurisprudencia tendrá serias implicaciones legales.

\section{SU-337, del 12 de mayo de 1999 \\ https://www.corteconstitucional.gov.co/relatoria/ 1999/su337-99.htm}

Magistardo ponente: Dr. Alejandro Martínez Caballero

Hechos que llevaron a la sentencia: menor de 8 años de edad a quien se asignó sexo femenino en el momento del nacimiento (parto atendido por partera), sin que se identificara alteración genital. A la edad de 3 años, durante un examen pediátrico, le encontraron genitales ambiguos, y fue diagnosticada con "seudohermafroditismo masculino", y se describió un falo de $3 \mathrm{~cm}$, pliegues labioescrotales con gónadas simétricas de $1 \mathrm{~cm}$ de diámetro, orificio único en el periné, "debido a un problema de trastorno en la síntesis de la testosterona”. El equipo médico que le valoró recomendó la readecuación genital por medio de la extirpación de las gónadas, clitoroplastia y vaginoplastia. Para la época en la que se radicó la tutela, la paciente tenía 7 años. Los médicos del caso se negaron a practicar la intervención quirúrgica, considerando que la decisión, ajustándose a la sentencia T477/95, debía ser tomada por la menor, y no por su madre. 
Por tal razón, la madre, quien ejerce la patria potestad sobre la menor, interpone la acción de tutela a fin de que se autorice la intervención quirúrgica. Según su criterio, y dado que la menor no podía tomar decisiones por sí misma, el hecho de esperar a que tuviera la capacidad para hacerlo alteraría su desarrollo psicológico, fisiológico y social. Atendiendo los hechos de este caso, le corresponde a la Corte Constitucional resolver lo siguiente:

Problema jurídico: ¿Los representantes de los menores pueden o no autorizar una intervención médica y quirúrgica destinada a readecuar los genitales de un infante, a quien le fue asignado un sexo masculino o femenino, después de habérsele diagnosticado alguna forma de ambigüedad sexual o genital, y que no esté de por medio del riesgo de muerte?

Análisis de la sentencia: se trata de una sentencia de unificación, y constituye un precedente judicial. La corte encuentra que, en los casos en los que una persona no goza de la suficiente autonomía para tomar libremente una decisión sobre un tratamiento específico, otras personas, en general los tutores o familiares, en virtud del principio de beneficencia, tienen el derecho y el deber de tomar las determinaciones necesarias para proteger la vida y la salud mediante el consentimiento sustituto, el que evidentemente también tiene unas limitaciones.

Anota la sentencia que "conforme a las actuales investigaciones académicas sobre la intersexualidad, la Corte no puede, ni le corresponde, establecer si la cirugía a temprana edad [...] es o no benéfica, por ende tampoco podemos decir que sea dañina, como parecen sugerirlo algunas de las críticas más radicales a estos tratamientos". Deja claro, después de investigar el concepto de muchas fuentes científicas nacionales e internacionales, que "existe un pluralismo médico para enfrentar la ambigüedad genital" con una evidente dicotomía frente al manejo quirúrgico. Ante esa circunstancia de duda sobre la pertinencia de los procedimientos, plantea la posibilidad de dejar a la privacidad de la familia la capacidad de decidir, pero también anota que "existen evidencias fuertes de que los padres $[\ldots]$ tienen mucha dificultad para entender verdaderamente los intereses de sus hijos con ambigüedad genital", y que, en tales situaciones, "es perfectamente humano que las decisiones de los padres tiendan más a basarse en sus propios temores y prejuicios, que en las necesidades reales del menor", y que "los hijos corren entonces el riesgo de ser discriminados por sus propios padres".

En el caso presentado puntualmente, tres argumentos permiten a la Corte Constitucional pronunciarse: 1) la connotación de urgencia para los procedimientos propuestos no está presente; 2 ) por la edad de la paciente (8 años), "ya tiene conciencia clara de su cuerpo", por lo cual la cirugía puede ser percibida como una agresión; y 3) "ya ha adquirido un grado de autonomía, que merece una mayor protección constitucional". Establece entonces la corte que, en este escenario, no es legítimo el consentimiento sustituto de la madre para que sea operada, y que "las cirugías y los tratamientos hormonales deben ser postergados hasta que la propia persona pueda autorizarlos".
Se pregunta entonces la corte: “¿a qué edad se puede presumir que han ocurrido los cambios sicológicos que invalidan el consentimiento sustituto paterno en caso de ambigüedad genital [...]?; “ “ ¿hasta qué edad se debe esperar para que la niña pueda autorizar esas intervenciones quirúrgicas y hormonales?"

Al respecto de la primera pregunta, manifiesta la Corte que no le corresponde "dirimir esa[s] difíciles polémicas", pero que "a los cinco años un menor no sólo ha desarrollado una identidad de género definida sino que, además, tiene conciencia de lo que sucede con su cuerpo y posee una autonomía suficiente para manifestar distintos papeles de género y expresar sus deseos". La Corte concluyó entonces que, "mientras no se ofrezcan nuevas evidencias científicas que obliguen a reconsiderar el anterior análisis, a partir de los cinco años, no es constitucionalmente admisible el consentimiento paterno sustituto para la remodelación de los genitales".

Al respecto de la segunda pregunta, establece que “deberá conformarse un equipo interdisciplinario, que debe incluir no sólo profesionales de la medicina sino también un sicoterapeuta y un trabajador social, que deberán acompañar a la menor [...]y a su madre" y que "en cada caso concreto, corresponderá a los equipos interdisciplinarios realizar las pruebas pertinentes para evaluar si la persona goza de la autonomía suficiente para brindar un consentimiento informado", y que, si lo brindan sin que necesariamente hayan alcanzado la mayoría de edad, los grupos interdisciplinarios deben velar para que el consentimiento sea genuino, completamente informado, y cualificado.

Como decisión de esta sentencia, la corte no accede a las pretensiones de la madre, considerando que "corresponde a la menor tomar la decisión sobre su identidad sexual”, y, para proteger los derechos al libre desarrollo de la personalidad y a la igualdad de la niña, ordena conformar un grupo interdisciplinario que atienda su caso, le brinde apoyo psicológico y social, y defina cuando la paciente tiene la autonomía para dar su consentimiento.

Alrededor del consentimiento, establece que este debe ser:

1. Libre: la persona debe tomar su determinación sin coacciones ni engaños.

2. Informado: "debe fundarse en un conocimiento adecuado y suficiente de todos los datos que sean relevantes para que el enfermo pueda comprender los riesgos y beneficios de la intervención terapéutica, y valorar las posibilidades de las más importantes alternativas de curación, las cuales deben incluir la ausencia de cualquier tipo de tratamiento".

3. Cualificado: hace referencia a que no basta con entregar la información, sino que se debe establecer, mediante preguntas $\mathrm{u}$ otras estrategias, que la decisión es auténtica y basada en el entendimiento de lo que se ha informado, y permite que "sólo terminen enfrentando efectivamente los peligros las personas que verdaderamente querían hacerlo", y que han sopesado el riesgo y el beneficio. Evidentemente, cuanto más 
invasivo sea un tratamiento, más informado y más cualificado deberá ser el consentimiento.

4. Persistente: no basta que el consentimiento sea dado en una sola ocasión, sino que se requiere que el permiso sea reiterado e, idealmente, sea dado en momentos distantes del periodo de duelo que entraña el hecho de tener un hijo con ambigüedad genital, y que, en ese proceso, se pueda complementar la información y brindar respuestas a las inquietudes que surjan.

Evidentemente, esta sentencia establece los criterios del consentimiento, la invalidez del consentimiento sustituto para realizar remodelación de los genitales en pacientes mayores de 5 años de edad, y le da estructura y piso legal a los grupos interdisciplinarios que hoy día evalúan y manejan los pacientes con DSDs en nuestro país.

\section{T-551, del 2 agosto de 1999 \\ https://www.corteconstitucional.gov.co/relatoria/ 1999/t-551-99.htm}

Magistrado ponente: Dr. Alejandro Martínez Caballero

T-692, de 16 de septiembre de 1999 https://www. corteconstitucional.gov.co/relatoria/1999/t-692-99.htm

Magistrado ponente: Dr. Carlos Gaviria Díaz

Hechos que llevaron a la sentencia: paciente de 2 años con hiperplasia suprarrenal congénita, con evidencia de genitales ambiguos, a quien el endocrinólogo y el cirujano tratantes, desde el nacimiento, le ordenaron una operación de "remodelación de sus genitales" al cumplir los 2 años de edad. Al superar por varios meses la edad recomendada y sin que hubiese sido operada, el padre solicitó que se tutelaran los derechos fundamentales de su hija, que, por consiguiente, se ordenara la realización de la cirugía programada lo antes posible, y que se suministrara a la menor el tratamiento necesario para su efectiva recuperación. El juez de tutela, que conoce el caso, determinó que, en un término no superior a 48 horas, se dispusiera lo necesario para que se le practicara la cirugía recomendada (sin que se hubiese constatado si se había llevado a cabo un proceso adecuado de consentimiento), decisión que llega para revisión a la Corte Constitucional. Cuando la corte indaga sobre el estado de la paciente, encuentra que ya fue operada, y que se le realizó "remodelación de genitales ambiguos" con el consentimiento sustituto paterno, sin que se hubiese hecho "un cambio o reasignación de sexo", como lo informaron sus médicos tratantes.

Problema jurídico: ¿Era legítimo el consentimiento sustituto paterno para la realización de dicho procedimiento?

Análisis de la sentencia: la sentencia T-551, del 2 de agosto de 1999, hace una síntesis y reitera la doctrina constitucional sobre el consentimiento informado en casos de ambigüedad genital consignados en la sentencia SU-337, de 1999.

De acuerdo a esa doctrina, los padres podían autorizar el procedimiento, ya que, por la edad de la paciente, no se había superado el umbral de los 5 años a partir de los cuales pierde validez constitucional el consentimiento paterno sustituto, pero se debía demostrar que, si el proceso de consentimiento (informado, cualificado y persistente) hubiese sido adecuado, no se hubiese realizado un cambio o reasignación de sexo, sino una "simple remodelación de los genitales”. La preocupación de la corte recae en establecer si, teniendo en cuenta, por la información disponible en ese momento, que esas cirugías de remodelación genital podrían ser invasivas y riesgosas y con resultados variables, se podrían llevar a cabo con el consentimiento del representante legal del menor, o si se debía respetar la autonomía del paciente y esperar que la tuviera, para que tomara sus propias decisiones.

La corte entonces consideró que la única opción posible es que los padres puedan decidir siempre y cuando tengan toda la información posible para que puedan tomar una decisión que tenga como eje central los intereses reales de los menores. Deja claro que, en el proceso de consentimiento, es de vital importancia la cualificación del mismo, es decir, que se tenga certeza que los padres comprenden a cabalidad los riesgos de las terapias ofrecidas, su reversibilidad, y las otras posibilidades de tratamiento quirúrgico, incluyendo la de no realizar ninguna cirugía. Considera la corte que la misma comunidad médica debe establecer protocolos que garanticen el cumplimiento de todos los requerimientos del consentimiento, y que los padres tengan claridad que, en los casos de ambigüedad genital, no necesariamente la asignación de un sexo implica la obligatoriedad de realizar cirugías destinadas a reconstruir el aspecto de los genitales de manera inmediata, y que la autorización que se dé para realizar los procedimientos no surja de una sola entrevista, sino que se dé de manera reiterada y con intervalos suficientes de reflexión por parte de los padres (consentimiento persistente y por etapas), y que, por supuesto, cumpla la formalidad de dejarlo por escrito.

En la sentencia T-692, de 16 de septiembre de 1999, en un caso clínico similar al anterior, se revisan los conceptos enunciados en la sentenciaT-551, y destacamos que la corte consideró que: “...en ciertos casos, la ambigüedad genital se encuentra asociada a amenazas graves a la salud física o la vida de la persona.", circunstancia en la que no habrían cuestionamientos éticos ni jurídicos para que los padres autoricen los procedimientos; pero que también surge un problema constitucional en aquellos casos en que la ambigüedad genital no se encuentra ligada a ninguna condición grave que ponga en riesgo la vida o la salud, y los médicos consideran la realización de cirugías de readecuación genital. Deja claro la corte que, ante las evidencias encontradas a favor y en contra de este tipo de procedimientos de remodelación genital en menores de 5 años, dejan a la privacidad de la familia la decisión al respecto siempre cuando se garantice y se verifique un proceso adecuado de consentimiento.

Esta sentencia plantea una situación en la que son claras las indicaciones clínicas para hacer la remodelación genital, puesto que no se está haciendo un cambio o reasignación de sexo, sino una adaptación para mejorar la ambigüedad genital y corregir posibles complicaciones (como hematocolpos). Estas modificaciones son válidas si cumple con el proceso de consentimiento informado, cualificado y persistente. Similar a este caso, puede suceder en pacientes 
con gónadas disgenéticas no palpables y la presencia del gen SRY, en los cuales es importante, por el riesgo de desarrollar neoplasias de hasta $50-60 \%$, realizar la remoción del tejido gonadal de manera temprana. ${ }^{7}$

\section{T-1.390, de 12 de octubre de 2000 \\ https://www.corteconstitucional.gov.co/relatoria/ 2000/t-1390-00.htm}

Magistrado ponente: Dr. Alejandro Martínez Caballero

Hechos que llevaron a la sentencia: al revisar la información fragmentaria del caso en la sentencia, se encuentra que se trata de un paciente de 3 meses de edad con evidencia de genitales ambiguos, y que al parecer incialmente es catalogado como un defecto hipospádico severo con asignación al sexo masculino. De los datos de la evaluación de endocrinología y cirugía pediátrica, se anota la solicitud de múltiples exámenes orientados a evaluar la anatomía interna y "determinar el sexo". La madre interpone una acción de tutela para que a su hijo se le realicen todos los exámenes pertinentes y se le ofrezca toda la atención necesaria, incluyendo su manejo quirúrgico. El juez falla a favor de la madre, ordenándole a su entidad promotora de salud (EPS) que se realice todo lo solicitado.

En evaluaciones posteriores, se refiere el hallazgo de sus genitales más como ambiguos que como los de un defecto hipospádico. En una evaluación de medicina legal, se anota: "diagnóstico de genitales ambiguos, con informe de un testículo y configuración cromosómica femenina. [...] Se informa además hipospadia[s] severa". En la revisión de la sentencia por parte de la Corte Constitucional, la madre indica "que su hijo es en realidad de sexo femenino", quedando pendientes la realización de exámenes, de cirugía y de la modificación del registro civil en el que figura como niño. Finalmente, el servicio de endocrinología informa que "ya definió que por exámenes hormonales hay presencia de gónada femenina y por cariotipo XX, el sexo genético del paciente es: FEMENINO", y se plantea la posibilidad de corregir la "hipertrofia de Clítoris y el seno Urogenital".

Problema jurídico: ¿Es o no procedente que, por vía de tutela, el juez constitucional ordene a una entidad de seguridad social, en este caso, el Instituto de Seguros Sociales (ISS), adelantar una cirugía de remodelación de los genitales, la cual ha sido recomendada por los especialistas como esencial para asegurar un desarrollo psicológico y físico satisfactorio de un menor que presenta una forma de ambigüedad genital?

Análisis de la sentencia: reitera la corte la doctrina constitucional consignada en la SU-337/99, y advierte que, si el procedimiento de remodelación genital no ha sido realizado, el juez de tutela de primera instancia debe comprobar si la autorización paterna reúne las características de "consentimiento informado cualificado y persistente", "pues de no ser así, el permiso sustituto no se adecua a la Carta, y mal podría ordenarse por vía judicial la práctica de una intervención médica que no contaba con un consentimiento informado válido, que es requisito constitucional esencial para todo tratamiento médico".
Aunque la sentencia no aporta elementos de análisis adicionales, demuestra con claridad las grandes dificultades que pasan los pacientes con DDSs y sus familias, con asignación de género inadecuada, problemas administrativos para la realización de sus estudios, inconsistencia en los conceptos, tanto médicos como jurídicos, en la información brindada por los médicos tratantes, y la evidente ausencia de valoración por un grupo multidisciplinario.

\section{T-1.025, de 27 de noviembre de 2002:}

https://www.corteconstitucional.gov.co/relatoria/ 2002/T-1025-02.htm

\section{Magistrado ponente: Dr. Rodrigo Escobar Gil}

Hechos que llevaron a la sentencia: se trata de un menor con ecografías antenatales que informaban feto de sexo femenino, pero que, al momento del nacimiento, se le asigna el sexo masculino por el hallazgo de un pene aparentemente normal, pero sin que se identificara en sus valoraciones iniciales la ausencia de gónadas palpables. Cerca a cumplir los 3 años, se hace diagnóstico de pubertad precoz, y se hace evidente la ausencia de gónadas palpables. A pesar de que se recomienda en ese momento valoración por endocrinología pediátrica, dicha valoración sólo es posible hasta completar los 4 años de edad. Se plantea en ese momento la posibilidad de que se trata de una paciente femenina severamente virilizada por una hiperplasia suprarrenal congénita, $y$, a los 4,5 años, se realiza cariotipo reportado $46, \mathrm{XX}$, el que confirma el diagnóstico.

Por los hallazgos, es presentado en una junta de pediatría y cirugía pediátrica, y se describe: "pene de $8 \mathrm{cms}$. y vello púbico con un desarrollo Tanner III.” El grupo tratante considera que, por la edad del paciente y sus hallazgos, debe continuar en el sexo masculino, y que se le debe ofrecer laparoscopia para resección de los genitales internos femeninos y cirugía genital ("moldeamiento de pene", en caso de ser necesario), con posterior suplencia hormonal masculina y colocación de prótesis testiculares. Dejan constancia que "existe un impedimento legal de acuerdo con la Corte Constitucional que no permite cirugías para 'cambio de sexo' sin el consentimiento de la persona hasta que tenga uso de razón", y se anota adicionalmente que, según el concepto de los padres, el paciente "padece de retardo mental y sicomotriz en un $60 \%$ ", afirmación que no es sustentada en un concepto de medicina legal que sólo detecta alteraciones del aprendizaje. Los padres interponen una acción de tutela para que el paciente sea operado, y solicitan la inaplicación de la Doctrina de la Corte Constitucional "en relación con el requerimiento del consentimiento informado del menor para la práctica de la cirugía de asignación de sexo [...], dadas las condiciones particulares del caso". El juez que conoce del caso considera que, como no existe un evidente riesgo que afecte la vida del paciente si la cirugía no es realizada, no es posible que los padres autoricen dicha intervención, y que se debe realizar, mediante un grupo interdisciplinario, un acompañamiento al paciente y su familia, y este mismo grupo debe establecer cuando el 
paciente tiene la autonomía para dar su consentimiento para que los procedimientos se realicen.

Problema jurídico: ¿Es procedente el consentimiento sustituto para la realización de la asignación de sexo, o, por el contrario, como se trata de un menor que ha sobrepasado el umbral de los 5 años, habría que esperar a que adquiera la madurez suficiente para adoptar por sí mismo dicha decisión?

Análisis de la sentencia: después de reiterar su doctrina, la Corte Constitucional introduce un concepto adicional: el consentimiento asistido. $\mathrm{Al}$ respecto, refiere que conforme con la evolución de las facultades del menor, se podría, mediante la participación de los padres o representantes legales de los mayores de 5 años, adoptar decisiones sobre la práctica de cirugías de asignación de sexo siempre y cuando: “(i) exista un acuerdo médico en torno a la alternativa clínica adecuada para el menor; y (ii) siempre que la identidad de género del infante se encuentra marcada o acentuada social y psicológicamente."

El objetivo sería evitar que se pospusieran cirugías que pudiesen ser necesarias hasta una edad cercana a la pubertad, con los efectos psicológicos traumáticos derivados de la postergación, pero deja la Corte claro que la aplicación del "consentimiento asistido no puede conducir al desconocimiento del consentimiento informado del menor (consentimiento asistido coadyuvado por la voluntad expresa del menor). Expresa entonces la Corte que, cuando el menor haya superado el umbral de los 5 años y, por retardo en su proceso de atención, no se haya hecho el manejo, "no necesariamente opera la regla de exclusión", y no se puede imponer como requisito único el consentimiento informado del menor, por lo cual se podría contar con la posibilidad del consentimiento asistido. Dice la corte: un "menor [...] entre los 6 y 7 años goza de un cierto grado de discernimiento y de madurez que le permite consentir en una operación de tal magnitud", y que: “En este orden de ideas, y en aplicación de los parámetros previstos por esta Corporación, es claro que los llamados a velar por la procedencia del consentimiento asistido que comporta el consentimiento prestado por los padres coadyuvado por la expresa voluntad del menor, son los profesionales de la salud, obviamente, destinando su lex artis a la defensa y protección de la autonomía e integridad del infante". Esto obliga a que: "El actuar de dichos profesionales de la salud deba ser lo más neutral y objetivo posible, alejado de criterios de conveniencia médica que alteren su imparcialidad". Concluye que: "Antes de los cinco años se debe proceder con base en la regla general del consentimiento sustituto, después, sólo con fundamento en el consentimiento informado del menor, a menos que, en atención a las particularidad [sic] de cada caso se disponga de una opción distinta, como el consentimiento asistido", pero siempre que haya "situaciones de excepción que legitimen la procedencia del consentimiento asistido o, en caso contrario, que reafirmen la salvaguarda del consentimiento informado del menor".

Se anota adicionalmente: "Por eso, en ningún momento, la Corte ha afirmado categóricamente que en torno a los estados 'intersexuales' o 'hermafroditismos' después de los cinco años sólo sea admisible el consentimiento informado del menor ya que, en ciertos casos, el carácter menos invasivo de la práctica médica o la ausencia de afectación a la autonomía del infante, otorgan relevancia al consentimiento sustituto o a la modalidad asistida como proyección del consentimiento del infante orientado a futuro". Aclara la sentencia que hace referencia a casos en los que no son forzosas las remodelaciones de los genitales externos, y pone como ejemplo la colocación de prótesis testiculares.

El concepto final importante es que la corte considera que son los equipos interdisciplinarios los que deberían establecer la pertinencia del consentimiento asistido o el respeto a la autonomía del paciente, esperando que tenga capacidad para dar su consentimiento.

Entonces, la procedencia del consentimiento asistido tiene varias exigencias:

1) Por tratarse de operaciones y tratamientos clínicos sumamente complejos, es necesario que se integre un equipo transdisciplinario de profesionales de la salud, para que realicen los estudios, diagnósticos y evaluaciones necesarias con el fin de proporcionar la asistencia científica más adecuada para preservar la salud integral del menor, teniendo en cuenta todos los aspectos físicos y psicológicos.

2) Que exista un consenso médico en torno a la alternativa clínica adecuada para el menor, y que dicha determinación se ajuste al principio de beneficencia.

3) El consentimiento asistido debe ser siempre coadyuvado por la expresa voluntad del menor.

4) La decisión de los padres y del menor, en ejercicio del consentimiento asistido, debe adecuarse a las recomendaciones médicas.

En su resolución, esta sentencia ordena la conformación de un grupo transdisciplinario que asista, oriente y asesore al menor y a su familia en el proceso de toma de decisión de la práctica de la cirugía de asignación de sexo, y que, cuando tengan toda la información disponible, consulten al menor y a sus padres sobre la decisión adoptada. Si la decisión es afirmativa y coincide con la del grupo transdisciplinario, ordena que el procedmiento se realice en los quince días siguientes a la manifestación de la voluntad de ser operado. Además, considera que, si no hay coincidencia entre los padres y el paciente o con el grupo transdisciplinario, el procedimiento no puede ser realizado.

A nuestro juicio, esta sentencia deja nuevamente en evidencia el papel preponderante del grupo transdisciplinario en la atención de los pacientes con DDSs, pero asigna también una gran responsabilidad, que obliga a que el actuar médico sea realmente objetivo y responsable, y siempre orientado al beneficio del menor.

6. T-1.021, de 30 de octubre de 2003 https://www. corteconstitucional.gov.co/relatoria/2003/t-1021-03.htm Magistrado Ponente: Dr. Jaime Córdoba Triviño

Hechos que llevaron a la sentencia: paciente de 9 meses de edad, en quien, "desde el momento de su nacimiento sus 
órganos genitales tenían una extraña morfología". Su madre instaura una acción de tutela, dado que no se le ha autorizado la realización del cariotipo ordenado por su médico tratante. Extrayendo la información médica citada de manera fragmentaria en la sentencia, se entiende que los genitales tenían el aspecto de una "hipospadia mediopeneana", que el reporte del cariotipo fue 46, XX (realizado en 3 oportunidades), que había una gónada derecha en "escroto", cuya histología por biopsia fue testículo, con una uretra masculina "sin presencia de vagina y de seno urogenital", y con un diagnóstico de hermafroditismo verdadero (hoy, DDS ovotesticular) que no queda demostrado, pues realizó exploración abdominal "encontrando un mínimo resto mulleriano [sic] en anillo inguinal izquierdo, vaso gonadal atrófico izquierdo, no se identificó útero, trompas de falopio o gónadas intrabdominalaes". Dicho remanente fue resecado, y el reporte anatomopatológico menciona que no se identifican restos müllerianos ni tejido gonadal. Adicionalmente, se le realizó corrección del defecto hipospádico con uretroplastia (por la técnica de Snodgrass) y corrección de curvatura ventral por la técnica de Nesbit, y se describió "cuerpo cavernoso atrófico en su tercio distal, cuerda ventral severa, glande pequeño y cuerpos cavernosos presentes".

Problema jurídico: ¿Fueron cumplidos los requisitos que la jurisprudencia constitucional ha fijado para el consentimiento sustituto de los padres en la autorización de intervenciones quirúrgicas relacionadas con la ambigüedad genital o estados intersexuales de menores de edad?

Análisis de la sentencia: en la sentencia, la corte hace un recuento de las reglas jurisprudenciales que se han fijado en fallos anteriores y que ya han sido ampliamente descritas, y hace énfasis en el consentimiento libre, informado, cualificado y persistente y en las implicaciones del consentimiento sustituto en los menores de 5 años. En el caso en concreto, se demuestra que era válido el consentimiento sustituto de los padres, dado que, para la edad del procedimiento, el paciente tenía 2 años, y que se cumplió adecuadamente con las exigencias del consentimiento informado sin que se hubieran vulnerado los derechos fundamentales a la identidad sexual, a la autonomía personal, y al libre desarrollo de la personalidad.

Se desprende de esta sentencia, para evitar problemas legales, la importancia de que se deje siempre constante en la historia clínica que se ha realizado un adecuado procedimiento de consentimiento, y que quede siempre consignado el contenido de la información brindada, incluyendo todas las alternativas posibles, que se ha cualificado el entendimiento de la información suministrada, que se han resuelto dudas, y que sea evidente que la autorización para el procedimiento fue otorgada de manera recurrente, y no sólo como el producto de una entrevista aislada con el paciente y su familia.

\section{T-912, de 18 de septiembre de 2008 https://www.corteconstitucional.gov.co/relatoria/ 2008/t-912-08.htm \\ Magistrado ponente: Dr. Jaime Córdoba Triviño}

Hechos que llevaron a la sentencia: menor de 5 años de edad con diagnóstico de "hermafroditismo verdadero" (hoy, DDS ovotesticular). Aunque en la sentencia no hay datos específicos del examen físico genital, se entiende que hay una virilización importante, pues el padre anota que "tiene los genitales masculinos completos". La biopsia de la gónada derecha es testículo atrófico, sin que se aclare su localización, y la izquierda es intraabdominal, con biopsia compatible con ovario. El paciente está registrado con género masculino, su identidad y rol de género son netamente masculinos. El padre instaura una acción de tutela para que su hijo sea operado y se haga adecuación al sexo masculino, pero sus médicos tratantes manifiestan la imposibilidad de este manejo por la edad del paciente, y porque, en su concepto, "los exámenes determinan que internamente, cromosómicamente y genéticamente, se trata de un paciente femenino". Según se anota, su cariotipo es $46, \mathrm{XX}$, su testosterona es muy baja (pero aparentemente evaluada en un momento de la vida en que la testosterona es normalmente baja), y, en una uretrocistografía, se describe "uretra de APARIENCIA FEMENINA de calibre y curso normal".

Problema jurídico: en el presente caso, corresponde resolver si resulta viable que la intervención requerida para la asignación de sexo se realice a partir del consentimiento sustituto de los padres, o si, por el contrario, como se trata de un menor que ha sobrepasado el umbral de los 5 años, se hace indispensable, de conformidad con la línea jurisprudencial fijada por la corte, esperar a que adquiera la madurez suficiente para adoptar por sí mismo dicha decisión.

Análisis de la sentencia: sostiene la corte que, en este caso, no es procedente el consentimiento sustituto, dado que el niño ya había superado el umbral de los 5 años, y es a él a quien le corresponde dar el consentimiento. Por haber superado esta edad, se plantea en este caso la posibilidad del consentimiento prestado por los padres (asistido) coadyuvado por la voluntad expresa del menor y apoyados por un grupo transdisciplinario que los acompañe en el proceso de decisión. En la resolución de esta sentencia, se ordena la integración de "un equipo transdiscplinario conformado por médicos (cirujanos, urólogos, endocrinólogos, pediatras y psiquiatras), psicólogos y trabajadores sociales, con el fin de que asistan, orienten y asesoren al niño $[\ldots]$ y a sus padres en el proceso de toma de decisión de la práctica de la cirugía de asignación de sexo". Establece que, una vez se haya brindado toda la información posible al paciente y a sus padres sobre las consecuencias de las cirugías y tratamientos para la asignación de género, se les debe consultar formalmente sobre la decisión adoptada al respecto del procedimiento quirúrgico. Deja claro que, si la decisión es afirmativa y coincide con el concepto del grupo interdisciplinario, la cirugía debe ser hecha en los quince días siguientes a la manifestación de la voluntad, pero que si no hay coincidencia entre el paciente y sus padres, o si la decisión del paciente y sus padres no coincide con la del grupo interdisciplinario, la cirugía no podrá ser realizada, y sólo se podrá llevar a cabo posteriormente si en algún momento hay coincidencia en la decisión. 
Evidentemente, en este caso, la única decisión que no se podría tomar es la de realizar una genitoplastia feminizante y asignación al género femenino. A nuestro juicio, tendría cabida la realización de la corrección del defecto hipospádico dejando las gónadas in situ, y, más adelante, con la autonomía del paciente, se podrían tomar decisiones adicionales. Las cirugías ablativas, en este escenario, deberían evitarse, por su irreversibilidad en pacientes cuya identidad de género podría cambiar en el tiempo o no ser predecible.

\section{T-450A, de 16 de julio de 2013 https://www.corteconstitucional.gov.co/relatoria/ 2013/t-450a-13.htm}

Magistrado ponente: Dr. Mauricio González Cuervo

Hechos que llevaron a la sentencia: nace un bebé vivo de presunto sexo femenino. De acuerdo con la historia clínica, se advierte que, en el ítem "Datos del recién nacido", el género se denominó masculino; sin embargo, más adelante, en una anotación, se refiere a que el sexo es femenino. Según la accionante, la médica rural que atendió el parto no pudo determinar que se trataba de un bebé intersexual o ambiguo, y le informó a la madre que había nacido una niña, aunque posteriormente ésta notó que se trataba de un bebé con genitales ambiguos. "En efecto, a la madre le fue entregado el Certificado de Nacido Vivo con logotipos del Ministerio de la Protección Social y el DANE [Departamento Administrativo Nacional de Estadística], sin diligenciar la casilla de "sexo del nacido vivo", sin nota marginal ni ninguna observación."

"En el momento de presentar el certificado de nacido vivo a la Registraduría del Estado Civil del Municipio [...], se le informó al padre que el bebé [...]no podía ser registrado porque los protocolos de formato de registro civil de nacimiento, [sic] tienen como base el certificado de nacido vivo que indica el sexo femenino o masculino. Como éste no estaba definido, no estaba permitido diligenciar el registro civil de nacimiento. Cuando el padre del bebé solicitó a la funcionaria del Registro que colocara sexo femenino en el espacio correspondiente, ésta se negó a hacerlo argumentando que la norma no lo admitía."

Adicionalmente, "Debido a algunos problemas de salud del bebé, éste fue llevado [...] [a su EPS] para inscribirlo en el Sistema de Seguridad Social Subsidiada, pero en un principio se le negó el acceso al mismo por no contar con registro civil de nacimiento. Como consecuencia de lo anterior, se acudió al Comisario de Familia del Municipio, quien ordenó de manera inmediata el restablecimiento de los derechos del bebé".

Problema jurídico: "Si desconoce el derecho al registro y a la personalidad jurídica, la no inscripción de un niño cuyo certificado de nacido no precisa el sexo."

Análisis de la sentencia: "la Sala considera que de ninguna manera puede la indeterminación del sexo convertirse en un obstáculo para el ejercicio del derecho a la personalidad jurídica, el cual es inherente al ser humano por el solo hecho de existir. Atendiendo al principio de dignidad humana y al derecho a la igualdad, no existe razón que justifique que bebés y niños cuyo sexo no pueda ser identificado al nacer, no sean registrados y permanezcan ocultos frente al Estado y la sociedad. [...] Por consiguiente, las autoridades están en la obligación de registrar a los menores intersexuales o con ambigüedad genital. La decisión sobre la asignación del sexo en el registro civil de nacimiento depende de la decisión del equipo médico interdisciplinario de expertos. Las opciones de asignación de sexo en el registro civil para los intersexuales incluyen femenino, masculino o una anotación en un folio aparte conforme a lo señalado en la parte motiva de esta sentencia. El legislador regulará todo lo concerniente al registro de los menores intersexuales."

Es de aclarar que, a raíz de lo anterior, la Registraduría Nacional modificó "la inscripción de menores intersexuales o con genitales ambiguos cuando la asignación de sexo no corresponda a las categorías de femenino o masculino, disponiendo su consignación en un folio diferente que se suprimirá cuando se asigne definitivamente el sexo. Para lo anterior se requerirá de diagnóstico médico de intersexualidad o ambigüedad genital y autorización escrita del menor o de los padres" con el fin que no constituya un obstáculo para el cumplimiento de los derechos.

\section{T-622, de 28 de agosto de 2014}

https://www.corteconstitucional.gov.co/relatoria/

\section{4/t-622-14.htm}

Magistrado ponente: Dr. Jorge Ignacio Pretelt Chaljub

Hechos que llevaron a la sentencia: la información médica sobre el caso es fragmentaria. Se trata de un paciente cuya acción legal se instaura a la edad de 11 años. Se anota que al momento del nacimiento le asignan el sexo femenino sin que sus genitales fueran normales ya que el pediatra les informó que la menor presentaba "hiperplasia suprarrenal congénita", sin que ese diagnóstico se hubiese demostrado. A los 5 años, los padres le cambian el nombre femenino por uno masculino, porque "notaron que el desarrollo de sus facciones y comportamientos eran masculinos". A pesar que el nombre fue cambiado en el registro civil, en la tarjeta de identidad del paciente, al momento de entablar la acción legal, sigue apareciendo el sexo femenino. La madre manifestó que nunca consultó al médico ni le realizaron estudios. Al momento de la valoración inicial por medicina general a los 11 años se anota: "GENITALES AMBIGUOS CON PRESENCIA DE LABIOS MAYORES E IONTROITO [SIC] VAGINAL PEQUEÑO + MICROPENE. NO PALPO TESTÍCULOS". Adicionalmente, en una entrevista en una comisaría de familia, el paciente manifiesta: "estamos tramitando una operación porque tengo órganos sexuales femeninos y masculinos, pero yo quiero quedar con órganos masculinos porque yo me siento hombre y me siento atraído por las mujeres". El cariotipo realizado fue 46 , XY. Se anota también que se realizó ecografía abdominal, que "no identifica útero pero si (sic) identifica próstata"; en un examen de genética, se consigna: "Falo $3 \mathrm{~cm}$, sinus urogenital, criptorquidia, fusión labioescrotal", y, en un examen de cirugía pediátrica, se anota: "Región inguinal: No palpo gónadas. Genitales: Genitales ambigüos, falo angulado con huella meatal en glande, meato uretral hipospádico escrotal amplio, escroto bífido, vacío, hipotrófico". Con la información disponible, se define que 
se trata de un DDS XY y, dada la aparente ausencia de útero, se podría establecer que no se trata de una disgenesia gonadal, sino de un trastorno de la síntesis o de la producción de los andrógenos.

La madre en representación y nombre de su hijo solicita al juez de tutela que ampare sus derechos fundamentales a la dignidad humana, identidad sexual, personalidad jurídica, salud, vida digna y seguridad social, y, en consecuencia, se ordene a la entidad accionada brindar toda la atención integral al menor, incluyendo las cirugía de reasignación de sexo y los demás exámenes de procedimiento que se necesiten.

Problema jurídico: ¿Es procedente la cirugía de remodelación de genitales para el menor de 12 años?

Análisis de la sentencia: esta sentencia hace una revisión de la doctrina consignada en todas las sentencias previas que hemos revisado. Similar a los casos que hacen referencia las sentencias T-1.025/02 y T-912/08, se considera que no es válido el consentimiento sustituto, y se ordena la conformación de un grupo transdisciplinario para legitimar el consentimiento asistido coadyuvado por el menor, como se explicó claramente en las dos sentencias mencionadas.

Una vez más, este ejemplo demuestra el mal manejo de estos casos, la asignación de género precipitada sin evaluación, la inconsistencia en la consignación de los hallazgos del examen físico, la pobre evaluación del paciente, y las inmensas dificultades que afrontan los pacientes con DDSs en nuestro país.

\section{Discusión}

Este artículo se presenta como una guía para los lectores, en especial para el equipo médico y las entidades aseguradoras. Tal como lo muestran las sentencias proferidas por la Corte Constitucional, el manejo de los pacientes con DDSs debe ser proporcionado por un equipo transdisciplinario que garantice conceptos unificados, así como una línea de tratamiento integral y continua en las diferentes etapas de atención, bien sea pediátrica o en la adultez.

Esta evaluación transdisciplinaria es de vital importancia para ofrecer un abordaje integral a los individuos con DDSs y sus familias. ${ }^{2}$ Adicionalmente, poder incluir en el abordaje de estos pacientes personas expertas en derecho resulta importante para guiar el proceso de atención y definir las conductas más apropiadas bajo el marco legal colombiano.

Es importante entender que, los 5 años como límite para procedimientos quirúrgicos (entendiéndose estos como: reasignación, remodelación, adecuación, readecuación de los genitales, y trasformación de órganos sexuales) no es una directriz mandatoria, toda vez que de las diferentes sentencias analizadas se desprenden los siguientes conceptos, a saber:

1) Para analizar la procedencia de un consentimiento, es decir, si lo deben dar los padres o el menor, se deben tener en cuenta:

- la necesidad y/o urgencia del tratamiento;

- el impacto y/o riesgo del mismo; y

- la edad y/o madurez del menor.
2) El consentimiento sustituto de los padres de los menores de 5 años para las cirugías de remodelación de genitales es constitucionalmente válido, siempre $\mathrm{y}$ cuando haya cumplido las exigencias de ser informado, cualificado y persistente.

3) El límite de los 5 años establecido en algunas sentencias no es absoluto y, en cambio, se deben tener en cuenta las particularidades de cada caso. Evidentemente, puede ser admisible el consentimiento asistido coadyuvado por el menor mayor de 5 años para este tipo de procedimientos (sentencia T-1025/02), siempre y cuando sea clara la voluntad expresa del menor, y que las circunstancias que llevan al consentimiento asistido hayan sido discutidas ampliamente por el grupo transdisciplinario, que se demuestre el grado de autonomía del paciente que le permite participar en la decisión, que haya concordancia entre todas las partes $\mathrm{y}$, sobre todo, que el acto médico para la toma de la decisión quirúrgica sea lo más imparcial, objetivo y neutro posible, y que tenga como eje central el beneficio y la protección de los derechos del menor.

4)Ante cualquier duda sobre la voluntad expresa del menor mayor de 5 años para la realización de un procedimiento, o sobre el real beneficio del mismo, sería sensato por supuesto, si no hay una condición urgente, posponerlo hasta que el paciente tenga la autonomía para brindar su consentimiento.

5) Todo consentimiento (incluido el sustituto y el asistido) debe ser informado, cualificado y persistente.

6) Es necesaria la elaboración de un protocolo de consentimiento que permita avalar su calidad. En lo referente a "informado", debería consignarse toda la información suministrada con sus respectivas fechas, tocando todos los tópicos pertinentes, como son indicaciones del procedimiento, riesgos y complicaciones previsibles, implicaciones a largo plazo, alternativas de tratamiento, dejando constante si se planteó como posibilidad la de no realizar tratamiento quirúrgico, con sus respectivas implicaciones. Al respecto de "cualificado", debería registrarse el proceso que permitió la cualificación de la información. Finalmente, al respecto de "persistente", deberían consignarse las oportunidades, con su respectiva fecha, en que los padres y/o el paciente se reafirmaron en su voluntad de ser operado.

Como queda establecido en la Doctrina Constitucional de todas esta sentencias, el grupo transdisciplinar es fundamental en la atención de los pacientes con DDSs, pues ofrece al paciente un enfoque integral. Este grupo debería contar al menos con: endocrinólogo pediátrico, neonatólogo, genetista, urólogo y/o cirujano pediatra, psiquiatra, psicólogo, trabajador social, y el profesional del área jurídica de la institución que atiende el caso.

Es evidente que nuestra realidad es muy compleja, pues, desafortunadamente, hay un gran desconocimiento sobre el tema entre nuestros médicos e incluso especialistas, que 


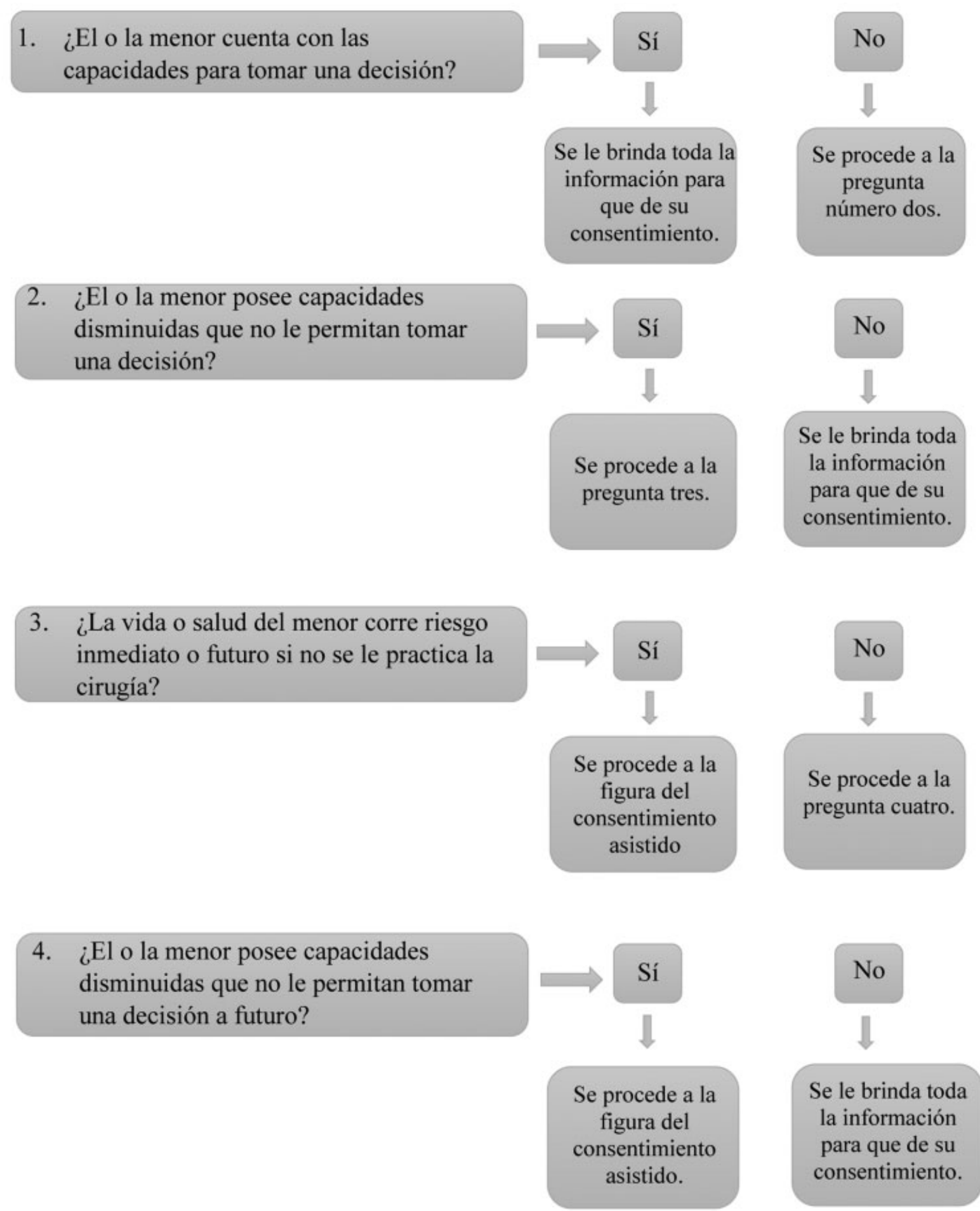

Fig. 1 Paciente mayor de 5 años con sugerencia de cirugía para readecuación de sexo.

conlleva errores frecuentes, como son la no identificación de la alteración genital, la descripción inadecuada de los genitales del paciente con ambigüedad, la asignación precipitada y errónea de género, la evaluación inadecuada, el inicio de medicamentos sin indicación, y la realización de procedimientos sin indicación clara y sin el aval de un grupo transdisciplinar. Para ensombrecer más la situación, el acceso al sistema de salud es difícil, descentralizado, la evaluación y el manejo de los pacientes es entorpecida por trámites administrativos engorrosos, y la consulta muchas veces es tardía, lo que lleva que, por ejemplo, la edad promedio de los pacientes que llegan por primera vez a la evaluación por nuestro grupo es mayor a 5 años. ${ }^{2}$ 


\section{Conclusión}

La toma de decisiones en pacientes con DDSs es un tema dilemático y complejo de abordar. La edad de 5 años no es un absoluto para no poder realizar intervenciones quirúrgicas a pacientes que sufran de DDSs. La realización de un proceso de consentimiento informado de manera adecuada permitirá apoyar las mejores decisiones para los pacientes y sus familias cuando la intervención se planea en edades por encima de los 5 años. Proponemos un flujograma que guiará el proceso de consentimiento de manera general (-Figura 1). Adicionalmente, por la naturaleza de las patologías, su diversidad y dinamismo, no es posible hacer recomendaciones globales a todos los casos que se presenten, incluyendo, en las consideraciones, el contexto social. Por lo anterior, nuestra recomendación, y como se enuncia previamente, la evaluación de estos pacientes debe ser de manera individual y utilizando el marco legal como guía, pero no como pauta inamovible.

Conflicto de Intereses

Los autores no tienen conflicto de intereses que declarar.

\section{Referencias}

1 Hutson JM, Grover SR, O'Connell M, Pennell SD. Malformation syndromes associated with disorders of sex development. Nat Rev Endocrinol 2014;10(08):476-487. http://www.ncbi.nlm.nih.gov/ pubmed/24913517 [Internet]

2 Fernandez N. Transdisciplinary Management of Patients with Disorders of Sex Development in Colombia. Limiting Factors for an Appropriate Management. Urol Colomb 2019;28(04):343-344

3 Kanhere M, Fuqua J, Rink R, Houk C, Mauger D, Lee PA. Psychosexual development and quality of life outcomes in females with congenital adrenal hyperplasia. Int J Pediatr Endocrinol 2015;2015(01):21. http://www.ijpeonline.com/content/ 2015/1/21 [Internet]

4 Meyer-Bahlburg HFL. Gender monitoring and gender reassignment of children and adolescents with a somatic disorder of sex development. Child Adolesc Psychiatr Clin N Am 2011;20(04):639-649. Doi: 10.1016/j.chc.2011.07.002 [Internet]

5 Romao RLP, Pippi Salle JL. Update on the surgical approach for reconstruction of the male genitalia. Semin Perinatol 2017;41 (04):218-226

6 Vellinga A, Smit JH, van Leeuwen E, van Tilburg W, Jonker C. Instruments to assess decision-making capacity: an overview. Int Psychogeriatr 2004;16(04):397-419

7 https://www.corteconstitucional.gov.co/relatoria/tematico.php? todos $=\% 25 \&$ sql $=$ ambiguedad + genital $\&$ campo $=\% 2 F \& p g=0$

8 Money J. Ablatio penis: normal male infant sex-reassigned as a girl. Arch Sex Behav 1975;4(01):65-71 